\title{
Application of Fourier Methods and Discrete-Cosinus Transformation in the Process of Processing of TV Images
}

\author{
Norinov M.U., Abdukadirov B.A., Gofurov M.R.
}

\begin{abstract}
This article describes the Fourier and discrete-cosine transform methods in the processing of $\mathrm{TV}$ images. The methods used in the processing of color and TV images, based on different physical effects, information processing technologies and hardware. The images obtained using these methods will have their own specifics. This, in turn, causes a wide variety of approaches both to the assessment of the quality of $T V$ images and to the processing of such images.
\end{abstract}

Keywords : Fourier series, Fourier transform, Fourier domain, wavelet transform, fast Fourier transform, discrete cosine transform.

\section{INTRODUCTION}

The Fourier transform is the basis of transformational image processing methods since the late 1950s, the use of a more modern transform, called the wavelet transform, simplifies the compression, transmission and analysis of many images. Unlike the Fourier transform, whose basic functions are harmonic functions, wavelet transforms are based on wavelet decomposition, called wavelets, of varying frequency and limited in time and space [2]. Such a decomposition for an image can be compared with a musical notation of a musical work, which indicates to the musician not only which note (frequency) to take, but also at what point - this should be done. In contrast, the usual Fourier transform contains only frequency information, time information is lost in the conversion process [4].

Any function that periodically reproduces its values can be represented as a sum of sines or cosines of different frequencies multiplied by some coefficients, usually this sum is called the Fourier series. The complexity of the behavior of the function does not matter. If only the function is periodic and satisfies easy mathematical conditions, it can be represented in the form of the above amount. At present, this statement is generally recognized, but at the time of its appearance it was a revolutionary idea, to the "getting used" to which the mathematicians of the whole world took more than a century.

Revised Manuscript Received on July 22, 2019

Norinov M.U., Abdukadirov B.A., Gofurov M.R.

Tashkent University of Information Technologies named after Muhammad Al-Khorezmi, Tashkent Uzbekistan, Fergana branch of the Tashkent University of Information Technologies named after Muhammad al-Khorezmi,Fergana

Uzbekistan, Fergana branch of the Tashkent University of Information Technologies named after Muhammad alKhorezmi,Fergana, Uzbekistan
At that time, the mainstay of mathematical thinking was the notion of regularity of functions [1]. From such positions, the idea that a complex function can be represented as a sum of simple (sines and cosines) seemed far from obvious. It is therefore not surprising that Fourier's ideas in this regard were met with skepticism. When a function is not periodic (but the area under its graph is finite), it can be expressed as an integral of sines or cosines multiplied by a certain weight function.

In this case, we deal with the Fourier transform, which in most practical problems turns out to be even more useful than the Fourier series. Both representations have an important characteristic feature [10]. The function defined by both the Fourier transform and the Fourier transform can be completely, without loss of information, restored (reconstructed) using some kind of reference procedure. This property is one of the most important properties of the considered representations, since it allows you to work in the "Fourier region" and then return to the original domain of the function definition without losing any information.

\section{THEORITICAL BACKGROUND}

Ultimately, it was precisely the effectiveness of the application of the apparatus of series and the Fourier transform for solving practical problems that turned it into a fundamental tool widely used and studied. Initially, Fourier's ideas were applied to solve the problem of heat propagation. This made it possible to present the differential equations describing the heat flux in a form that made it possible for the first time to obtain their solutions [6].

Fourier analysis methods provide clear and practical ways to study and implement a set of approaches for image enhancement.

\section{MAIN PART}

Being the basis of linear filtering methods, the Fourier transform provides considerable flexibility in the design and implementation of filtering algorithms in solving problems of image improvement, reconstruction and compression. The Fourier transform also lies in the foundation of many other important practical applications.

There are several types of Fourier transform:

1. A non-periodic continuous signal can be decomposed into

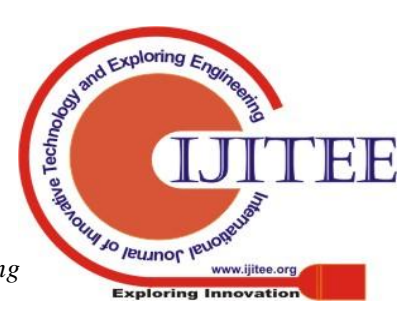


a Fourier integral;

2. A periodic continuous signal can be decomposed into an infinite Fourier series;

3. A non-periodic discrete signal can be decomposed into a Fourier integral;

4. A periodic discrete signal can be decomposed into a finite Fourier series.

A computer is able to work only with a limited amount of data, therefore, it is really able to calculate only the last type of Fourier transform. Consider it in more detail.

Let discrete signal $\mathrm{x}[\mathrm{n}]$ has a period $\mathrm{N}$ points. In this case, it can be represented as a finite series (i.e., a linear combination) of discrete sinusoids:

$$
x[n]=\sum_{k=0}^{N / 2} C_{k} \cos \frac{2 \pi k\left(\mathrm{n}+\varphi_{k}\right)}{N} .
$$

Equivalent recording (each cosine is decomposed into sine and cosine, but now without phase):

$$
x[n]=\sum_{k=0}^{N / 2} A_{k} \cos \frac{2 \pi k \mathrm{n}}{N}+\sum_{k=0}^{N / 2} B_{k} \sin \frac{2 \pi k \mathrm{n}}{N} .
$$

The algorithm for the inverse Fourier transform is obvious (it is contained in the formula of a Fourier series; to carry out the synthesis, you just need to substitute the coefficients in it). Consider the direct Fourier transform algorithm, i.e. finding coefficients Akand Bk.

System of functions

$$
\left\{\sin \frac{2 \pi k \mathrm{n}}{N}, \cos \frac{2 \pi k \mathrm{n}}{N}\right\}, k=0, \ldots, \frac{N}{2}
$$

from the argument $\mathrm{n}$ is an orthogonal basis in the space of periodic discrete signals with period $\mathrm{N}$. This means that to decompose any element of space (signal) through it, you must calculate the scalar products of this element with all the functions of the system and normalize the resulting coefficients. Then for the original signal the formula of the basis expansion with the coefficients Akand Bk.

So, the coefficients Akand Bkcalculated as scalar products (in the continuous case - integrals of the product of functions, in the discrete case - the sum of the product of discrete signals):

$$
\begin{gathered}
A_{k}=\frac{2}{N} \sum_{i=0}^{N-1} x[i] \cos \frac{2 \pi k i}{N}, \quad \text { npu } \quad k=1, \ldots, \frac{N}{2}-1, \\
A_{k}=\frac{1}{N} \sum_{i=0}^{N-1} x[i] \cos \frac{2 \pi k i}{N}, \quad n p u \quad k=0, \frac{N}{2}, \\
B_{k}=\frac{2}{N} \sum_{i=0}^{N-1} x[i] \sin \frac{2 \pi k i}{N}, \text { npu } k=0, \ldots, \frac{N}{2} .
\end{gathered}
$$

calculation of Fourier transforms requires a very large number of multiplications (about N2) and computing the sines. There is a way to do these transformations much faster: in about $\mathrm{N} \cdot \log 2 \mathrm{~N}$ multiplication operations. This method is called the fast Fourier transform.(БПФ). It is based on the fact that among the factors (sines) there are many repetitive values (due to the periodicity of the sine). The FFT algorithm groups the terms with the same factors, significantly reducing the number of multiplications. As a result, the FFT speed can be hundreds of times faster than the standard algorithm (depending on $\mathrm{N}$ ). It should be emphasized that the FFT algorithm is accurate. It is even more accurate than the standard one, since reducing the number of operations, it leads to smaller rounding errors.

However, most of the FFT algorithms have a feature: they are able to work only when the length of the analyzed signal $\mathrm{N}$ is a power of two. Usually this is not a big problem, since the analyzed signal can always be padded with zeros to the required size. The number $\mathrm{N}$ is called the size or length of the FFT.

For images representing a two-dimensional signal, the spectrum is also a two-dimensional signal. The basic functions of the Fourier transform are

$$
\mathrm{h}_{k_{1}, k_{2}}^{ \pm}\left(\mathrm{n}_{1}, \mathrm{n}_{2}\right)=\sin \left(\frac{2 \pi k_{1} \mathrm{n}_{1}}{N_{1}} \pm \frac{2 \pi k_{2} \mathrm{n}_{2}}{N_{2}}\right),
$$

and the phases may also be different. In the image, each of these basis functions represent a wave of a certain frequency, orientation, and phase.

HereN1 $\mathrm{xN} 2$ - the size of the original signal, it is the size of the spectrum, $\mathrm{k} 1$ andk2 - these are the numbers of the basis functions of the number of coefficients of the two-dimensional discrete wagon transform (DFT) at which these functions are located. Since the size of the spectrum is equal to the size of the original signal, $\mathrm{k} 1=0, \ldots, \mathrm{N} 1-1 ; \mathrm{k} 2=$ $0, \ldots, \mathrm{N} 2-1$.

$\mathrm{n} 1$ andn2 - argument variables of basic functions. Since the domain of definition of basic functions coincides with the domain of definition of a signal,atn1 $=0, \ldots, \mathrm{N} 1-1 ; \mathrm{n} 2=$ $0, \ldots, \mathrm{N} 2-1$.

The two-dimensional DFT in a complex form is defined by the following formulas (here $\mathrm{x}[\mathrm{n} 1, \mathrm{n} 2]$ - source signal as wellX[k1,k2] - its spectrum):

$$
\begin{aligned}
& \mathrm{X}\left[k_{1}, k_{2}\right]=\sum_{n_{1}=0}^{N_{1}-1} \sum_{n_{2}=0}^{N_{2}-1} x\left[n_{1}, n_{2}\right] \cdot 1^{-j n_{1} k_{1}\left(2 \pi / \mathrm{N}_{1}\right)} 1^{-j n_{2} k_{2}\left(2 \pi / \mathrm{N}_{2}\right)}, \\
& x\left[n_{1}, n_{2}\right]=\frac{1}{N_{1} N_{2}} \sum_{k_{1}=0}^{N_{1}-1} \sum_{k_{2}=0}^{N_{2}-1} X\left[k_{1}, k_{2}\right] \cdot 1^{j n_{1} k_{1}\left(2 \pi / \mathrm{N}_{1}\right)} 1^{j n_{2} k_{2}\left(2 \pi / \mathrm{N}_{2}\right)} .
\end{aligned}
$$

\section{Discussions}

Direct calculation of a two-dimensional DFT using the above formulas requires huge computational costs. However, it can be proved that a two-dimensional DFT has a separability property, i.e. it can be calculated sequentially from two dimensions. To calculate the two-dimensional DFT, it suffices to calculate the one-dimensional complex DFT of all the image lines, and then

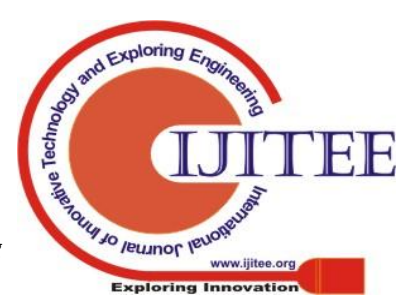


calculate the one-dimensional complex DFT of all columns in the resulting "image". In this case, the results of all one-dimensional complex DFTs should be recorded in the place of the initial data for these DFTs. For example, when calculating a one-dimensional DFT of the first row of an image, you need to write the result of the DFT into the first row of this image (it is the same size). To do this, each "pixel" should be stored as a complex number [5].

Thus, an effective algorithm for calculating the DFT of an image consists in calculating one-dimensional FFT first from all rows and then from all columns of the image [6].

For the spectra of generalized Fourier series of displaced blocks obtained from a certain realization of the signal, the coefficients were calculated and correlations were studied in [7]. The solution of the problem is given for both continuous signals and discrete signals given by a certain grid of samples. Analytical and numerical calculations of inter-block correlation with arbitrary shift between signal blocks for the most widely used for image and video compression of discrete orthogonal transformations are performed.

One of the discrete orthogonal transformations, which is widely used in the compression of digital images with losses, is the discrete cosine transform (DCT) [8].

DKP is a well-studied and highly efficient conversion proposed by B. Chen and used in JPEG, MJPEG, MPEG-1, MPEG-2, MPEG-4 formats. In fact, this method is similar to the two-dimensional discrete Fourier transform and differs from it only by the basic functions used. The advantage of DCT is the rapid convergence of the series, which provides less error in conversion.

Forward and reverse DCT are described by the following equations:

$F(u, v)=(1 / 4) C(u) C(v) \sum_{x=0}^{7} \sum_{y=0}^{7} p(x, y)\left[\cos \frac{(2 x+1) u \pi}{16}\right]\left[\cos \frac{(2 y+1) v \pi}{16}\right]$

$f(x, y)=\frac{2}{N} \sum_{u=0}^{N-1} \sum_{v=0}^{N-1} C(u) C(v) F(u, v) \cos \frac{(2 x+1) u \pi}{2 N} \cos \frac{(2 y+1) v \pi}{2 N}$, where $\mathrm{v}$ is the horizontal coordinate of the graphic block, $\mathrm{u}$ vertical, $\mathrm{x}$ - vertical coordinate inside the block, buty horizontal coordinate inside the block, $\mathrm{C}(\mathrm{u}), \mathrm{C}(\mathrm{v})=1 \sqrt{ } 2$ for $\mathrm{u}, \mathrm{v}=0$ and $\mathrm{C}(\mathrm{u}), \mathrm{C}(\mathrm{v})=1$, otherwise.

$$
A(u)= \begin{cases}\frac{1}{\sqrt{2}}, & \text { если } u=0 \\ 1, & \text { если } u \neq 0\end{cases}
$$

This method involves dividing the frame into blocks of 64 (8x8) samples, called signaling matrices. After that, the signal matrix is converted into a matrix of frequency coefficients of the same size, which can be considered as a two-dimensional spectrum of the image in the horizontal and vertical directions. Moreover, in such a matrix, the coefficients in the upper left corner correspond to the low-frequency component of the image, and in the lower right corner to the high-frequency component.

A feature of the DCT spectrum is that the main energy of the frequency components of this spectrum is concentrated in a small area near zero frequencies. The amplitude of the high-frequency components is either small or just zero, so only those frequency coefficients of the DCT matrix that exceed the accepted threshold values are transmitted. Coefficients below the threshold value are considered to be zero and zigzag reading and compression are not transmitted by the statistical compressor of long series.

\section{RESULTS}

If during transmission only zero coefficients are discarded, then compression is obtained without loss of quality, i.e. after decompression, the image will not be different from the original. However, the compression ratio is not high and on average is 10-20 times, depending on the detail of the image. To control the compression ratio, the DCT coefficients are divided into specific numbers (quantization matrix), followed by rounding to an integer, which increases the length of the chains of zero coefficients and, accordingly, the compression ratio. However, this rounding of data on the one hand leads to an increase in image compression, on the other hand, to irreversible loss of information, as a result of which, at large compression ratios, smoothness of brightness changes at the block boundaries is disturbed, which leads to distortions in the form of a block effect, which reduces intelligibility and quality of the restored image.

This method has good performance, combines well with the block method of motion compensation and provides good image quality at video stream speeds of more than $5 \mathrm{Mbit} / \mathrm{s}$. However, at lower speeds, the distortion in the form of a block effect is strongly pronounced, as a result of which the image takes on a mosaic look, which is the main disadvantage of this compression method.

DKP is used in JPEG and MPEG image compression standards.

The algorithm for quickly scaling images of video frames in real time using the discrete cosine transform (DCT) method during group video conferencing is presented in [13]. To avoid the execution of resource-intensive operations, the whole process of composition of the video stream is carried out by the memory area related to DCT. The application of the presented algorithm not only allows to increase the speed of computational processing, but also to improve the quality indicators of scalable video stream frames.

A promising direction to reduce the computational complexity of the discrete cosine transform is the identification of redundant computations and their removal is proposed in [10]. A new prediction method is proposed for the discrete cosine transform coefficient with quantized zeros for the effective implementation of intraframe video coding by identifying such redundant computation. The proposed method is based on two key ideas: the derivation of the bounds of the conversion coefficient and the intermediate signals of the Loeffler discrete cosine transform algorithm, and the selection of sufficient conditions for predicting the coefficient with quantized zeros.

Discrete cosine transform (DCT) for more than 30 years has been widely and 
successfully used in the field of encoding images and video data in the standards JPEG, MPEG-1/2/4 and H.264 [6]. It is reported on the results of studies in which it is shown that under certain conditions, DCT can be considered as a stable approximation of the Karhunen-Loeve transform (PCL, Karhunen-Loewe transform), the algorithms based on which have a significantly higher performance than the DCT algorithms. The theoretical possibility of developing new algorithms is substantiated, in which the best characteristics of both of these transformations are realized at the same time, which provides support for a stable approximation of PCLs and providing comparable or lower computational complexity than the DKT algorithms. For this purpose, an iterative algorithm is proposed with a cascade structure from a set of mathematical operations for calculating the FFT based on the multiplication of complex numbers (the graphic representation of each of these operations resembles butterfly wings).

Video compression methods based on three-dimensional discrete cosine transform as applied to video surveillance systems are discussed in [9]. To reduce the computational complexity, it is proposed to use a three-dimensional pseudo-cosine transform; it is implemented without multiplication operations; the quantization procedure is implemented without division operations.

A discrete cosine transform architecture is proposed, which allows to reduce power consumption while maintaining an acceptable peak signal-to-noise ratio when compressing images by changing process parameters and significantly increasing the supply voltage. The proposed architecture also makes it possible to predict a decrease in image quality for various computation paths and select the best one, while ensuring high image quality.

The coding scheme provides a significant improvement in the frequency of bit errors before the traditional image coding scheme based on the discrete cosine transform (DCT) [3]. The proposed scheme uses either traditional DCT or DSE type 7 discrete sine transform for all intraframe prediction modes of video encoding. It is noted that this approach is applicable to any intraframe predication scheme based on blocks in codecs that use transformations separately in the vertical and horizontal directions.

The mode selection algorithm based on the improved absolute difference conversion (SATD) for intraframe video encoding in the H.264 / AVC standard is performed in two stages. In the first stage, the low-frequency components of the macroblock discrete cosine transform blocks are used to select the prediction mode of $14 \mathrm{MB}$ or $116 \mathrm{MB}$, and in the second stage, the SATD coefficient, including the SATD values and their dispersion, are used to skip unsuitable modes for selecting the speed-optimized mode. The proposed algorithm is compared with some $\mathrm{k}$

One of the main technical problems of the implementation of the transcoder from the MPEG-2 Y.264 / AVC video coding standard is high computational complexity [5].

\section{CONCLUSION}

Empirical results show that the power consumption for the discrete cosine transform when decoding macroblocks of the MPEG-2 bitstream has a strong correlation with the intra-block prediction modes of the H.264 / AVC standard. Based on this relationship, a mode skip rule is proposed on the choice of the intra-block prediction mode in the coding part of the H.264 / AVC transcoder. This allows an average of $72.36 \%$ reduction in the computational complexity of the transcoder.

A simple and effective method for determining the magnitude of the "dead zone" with non-uniform quantization is proposed. It is shown. that such quantization provides a smaller amount of compressed quantized data than uniform quantization and quantization using the generalized Lloyd's algorithm. The relationship between the choice of quantization method and the efficiency of context-dependent coding of quantized coefficients of the discrete cosine transform, as well as the effectiveness of pre-filtering and post-processing to eliminate the block effect on the decoded images is considered.

\section{REFERENCES}

1. Berezkin E.F. Fundamentals of information theory and coding: Tutorial. M .: NIIUU MEPhI, 2010. - 312 page.

2. Gaiduk A.R. Continuous and discrete dynamical systems. -2 nd ed. Pererab.-M .: Educational-methodical and publishing center "Educational literature". 2004.- 252 page.

3. Egorov, V.A. Synthesis of an arrhythmic continuous-discrete controller for a linear object [Text] // Samar Bulletin. state tech. un-that. - Ser. Technical science. - № 3 (31). - Samara State Technical University: 2011. - pp.44-51

4. Zigler K. Methods of designing software systems. -M .: Mir, 1995

5. Kirillov S.N., Pospelov A.V. Discrete signals in radio systems. Tutorial Ryazan. RGRTA, 2003. 60page.

6. Kirillov, S.N., Vinogradov, OL, Lotsmanov, A.A. Algorithms for the adaptation of digital filters in radio devices. Tutorial. Ryazan. RGRTA, 2004. 80s.

7. Kobern A. Modern methods of describing the functional requirements for systems: Per. from English-M .: LORI, 2002

8. Lande D.V. Basics of information flow integration / D.V. Lande - K Engineering, 2006. - 240 seconds.

9. Fatrell, R., et al. Software Project Management: Achieving Optimal Quality with Minimal Cost / R. Fatrell, D. Schafer, L. Schafer: Translation from English. - M .: Williams, 2003.

10. Maltsev A.I. Algorithms and recursive functions. M. "Science", 1985. 